\title{
DRG and Other Problems
}

DRG - die Einführung dieses Abrechnungssystems wird die medizinische Versorgung und die Organisation im stationären Bereich in Deutschland gewaltig ändern. Dies ist sicher. „Aber - Ist dies ein sinnvolles System für die Gesamtmedizin? Die Zeit wird es zeigen. Jedenfalls werden nur diejenigen Kliniken die DRGs überleben, die mit dem neuen Abrechnungssystem optimal umgehen können“ - ohne Zweifel eine wichtige und sicher auch richtige Feststellung von Prof. C. Orfanos, Berlin, in der ersten Originalarbeit dieses Heftes. Prof. Orfanos beschäftigt sich in diesem Artikel mit den Auswirkungen und Konsequenzen der DRGs in unserem Fach, die er sehr strukturiert darstellt, an Beispielen verdeutlicht und klar anspricht. Verschiedene Möglichkeiten zur Verbesserung der Wirtschaftlichkeit unserer Kliniken sind herausgearbeitet. Sehr wichtig erscheint mir die Forderung aus Gründen einer ethischen Transparenz, dass „die unmittelbar behandelnden Ärzte von den erlösorientiert kodierenden Kräften im Krankenhaus getrennt agieren“. Das hilft, unsere „Kunden“ nicht vornehmlich nach „DRG-optimal zu managen“. Ein lesenswerter Artikel!

Die zweite Originalarbeit beschäftigt sich mit dem immer noch und immer wichtiger werdenden Thema der hypertrophen Narben und Keloiden, deren Behandlung nach wie vor schwierig bleibt. Die offene Studie verwendet Hansaplast Narbenreduktion, ein kostengünstiges Polyurethanpflaster. Verbesserungen wurden bei allen Patienten beobachtet bei zugleich problemloser Handhabung und sehr guter Verträglichkeit. Weitere Studien werden zeigen, ob sich die positiven Ergebnisse bestätigen lassen.

Schnell, ohne Zeitaufwand, bequem und schmerzlos soll sie sein, die Abheilung der Schuppenflechte, zumindest an den sichtbaren Hautregionen. Das ist der Wunsch vieler Patienten. Der ExcimerLaser (UVB $308 \mathrm{~nm}$ ) verspricht genau dieses. Wird noch zusätzlich Calcipotriol appliziert, nimmt die Zahl der Sitzungen bis zur „Remission“ deutlich ab. Dies jedenfalls berichten die Autoren B. Kardorff et al. in der vorliegenden Arbeit. Der Excimer-Laser erlaubt, das UVB exakt auf die Herde zu fokusieren, ohne Reizung der Umgebung und dadurch eine raschere Abheilung zu erreichen. Das ist offensichtlich durch Calcipotriol genauso zu verstärken wie bei konventionellen Phototherapien. Plaques von ca. $10 \times 10 \mathrm{~cm}$ lassen sich in ca. 20 Minuten behandeln, was die Limitierung der Flächenausdehnung klar macht, kaum mehr als 10 \% der Körperoberfläche sind in mehreren Sitzungen zu behandeln. Sicher erlauben die beschriebenen 8 Patienten noch keine Beurteilung des Effektes der Kombination Excimer-Laser und Calcipotriol. Klinische kontrollierte Studien im Seitenvergleich sind zu fordern und die Zeitverläufe sind zu prüfen.

Es schließen sich zwei interessante Kasuistiken an. Die erste stellt eine Familie mit Morbus Dowling-Degos mit der ungewöhnlichen ausschließlichen genitoperianalen Manifestation der retikulären Pigmentierungen vor. Die Autoren zeigen eindrückliche klinische und histologische Bilder und fassen das Krankheitsbild und die vielfältigen Differenzialdiagnosen kurz zusammen. Die zweite Kasuistik stellt eine Eosinophile Zellulitis (Wells-Syndrom) bei zwei Patienten vor, die nach Insektenstich bzw. Pneumonie bei Polychemotherapie wegen Mammakarzinom auftraten. Die Autoren betonen ausdrücklich die wahrscheinlich höhere Inzidenz als oft angenommen und die Differenzialdiagnose von urtikariellen, blasenbildenden Dermatosen, allergologische Reaktionen aber auch von therapieresistenten Erysipelen.

Es schließt sich ein Bericht (Zusammenfassungen der 7 Vorträge) über den zweiten Workshop: Dermatologische Labordiagnostik wissenschaftlich begründet, praxisrelevant und wirtschaftlich am 18.1.2003 in Leipzig an. Das Heft endet mit einem historischen Überblick - 75 Jahre Deutsche Gesellschaft für Lichtforschung, gegründet von Prof. Hans Meyer zur Vertiefung der Interdisziplinarität. Herr Prof. Jung gibt einen Überblick über die Entwicklung der Lichtbiologie vom Ende des 19. Jahrhunderts bis in die Gegenwart. In diesem Artikel gelingt es Herrn Prof. Jung, alle maßgeblichen Initiatoren und Förderer der Lichttherapie mit lesenswerten Einzelheiten darzustellen. Lichttherapien begannen im reizvollen, klimatisch begünstigten Bled, Slowenien. Die wichtige und anhaltende „Befruchtung“ der medizinischen Photobiologie aus den Basiswissenschaften Physik, Chemie, Immunologie, Physiologie. Ein lesenswerter, reich bebildeter Artikel. 